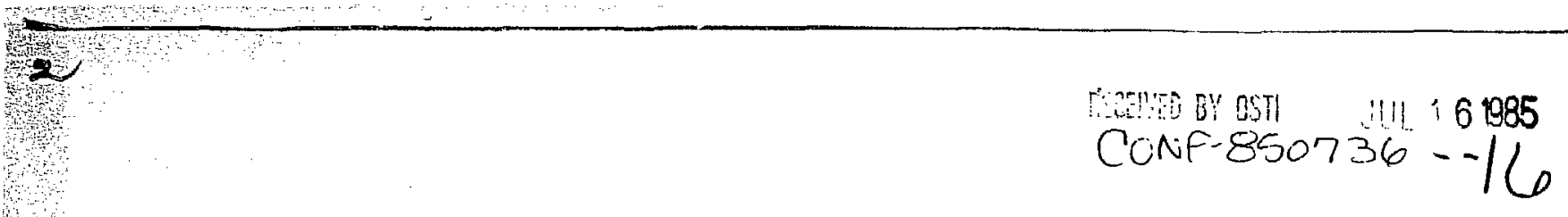

UCRL- 92231

PREPRINT

\title{
POSITRON ANNIHILATION AND PRESSURE-INDUCED ELECTRONIC s-d TRANSITION
}

A. K. McMahan

University of California

Lawrence Livermore National Laboratory

Livermore, California 94550

H. L. Skriver

Riso National Laboratory

DK-4000 Roskilde, Denmark

This paper was prepared for submittal to Proceedings of the APS Topical Conference on Shock Waves in Condensed Matter-1985

Spokane, Washington

July $21-26,1985$

June 1985

This is a preprint of a paper intended for publication in a journal or proceedings. Since changes may be made before publication, this preprint is made available with the understanding that it will not be cited or reproduced without the permission of the author.
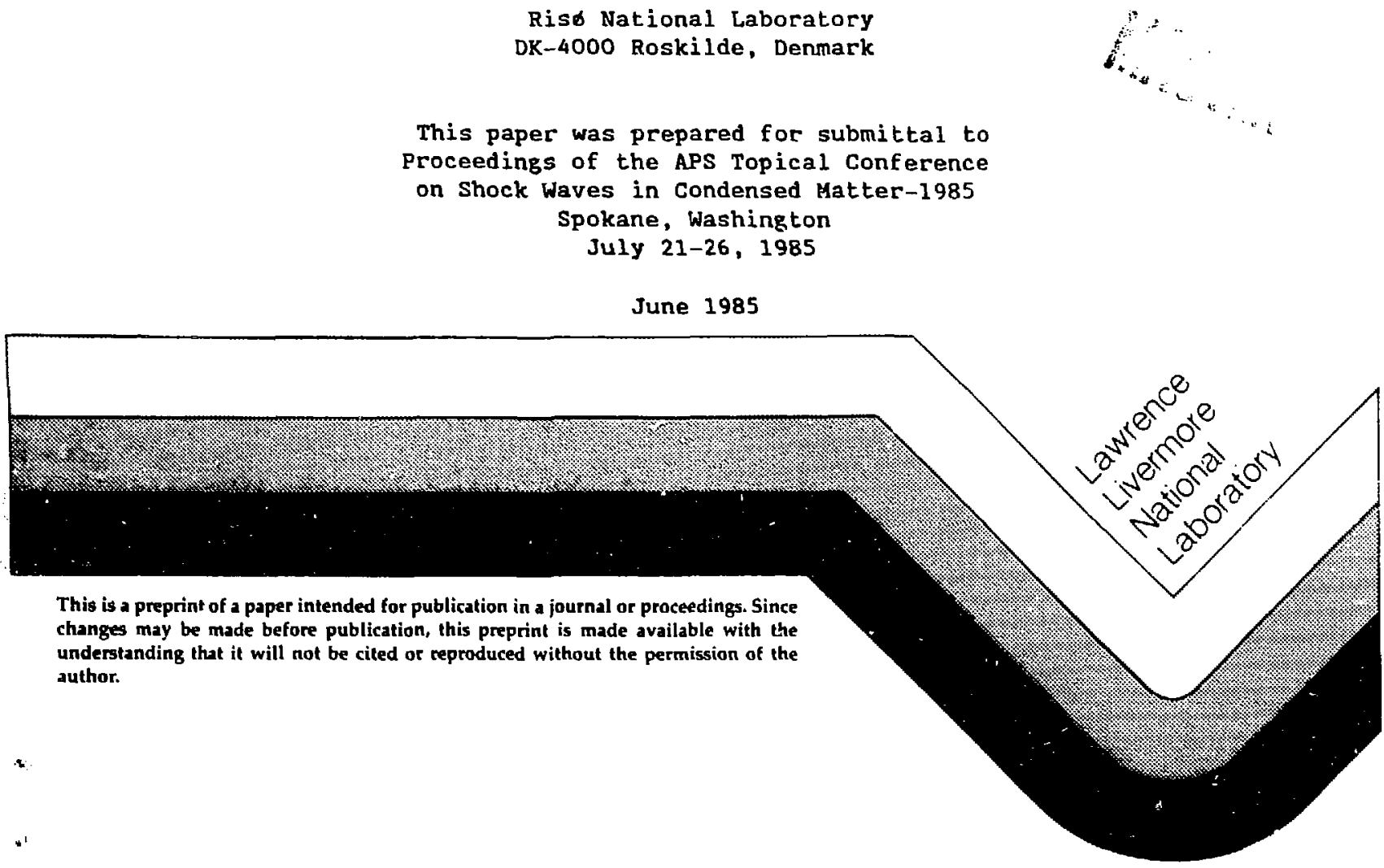
This repart wns prepared as an account of work sponsored by an agency of the United States Government. Neither the United States Government dor any agency thereof, nor any of their employes, makes any warranty, express or implied, or assumes any legal liability or responsibility for the accuracy, completeness, or usefulness of any information, apparatus, product, or process disclosed, or represents that its use would not inîinge privately owned rights. Reference herein to any specific commercial product, process, or service by trade name, trademark, manufacturer, or otherwise does not necessarily constitute or imply its endorsement, recommendation, or favoring by the United States Government or any agency thereof. The views and opinions of authors expressed herein do not necessarily state or reflect those of the United States Government or any agency thereof.

POSITRON ANIIHILATION AND PRESSURE-IMDUCED ELECTRONIC s-d TRANSITION

A. K. Mckahan

University of California

Lawrence Livermore National Laboratory

Livermore, California 94550

H. L. Skriver

Riso National Laboratory

DK-4000 Roskilde, Denmark

ABSTRACT

The polycrystalline, partial annihilation rates for positrons in compressed cesium have been calculated using the linear muffin-tin orbitals method. These results suggest that the pressure-induced electronic s-d transition in Cs should be directly observable by momentum sensitive positron annihilation experiments.

\section{INTRODUCTION}

Electronic s-d transition is a comnon phenomenon in high pressure physics, responsible for a variety of equation of state anomalies, insulator-metal transitions, and crysta:lographic phase transitions. 1 These are indirect manifestations of the pressure induced transfer of electrons from s-like (actually sp hybrid) valence states to those of predominately d character. We suggest here that positron annililation measurements can permit direct observation of the electron transfer itself. This follows from the fact that s-band valence electrons tend to occupy states near the center of the Brillouin zone, while the d states to which they transfer are located near the zone boundaries. Thus, techniques which probe quantities related to the electron momentum density will detect this transfer.

Positron annihilation is suited to high pressure experiments since the positron source $\left(e . \mathrm{g} . \mathrm{Na}^{22}\right.$ ) can be included in the pressure cell, while the annihilation $Y$ rays pass through, and are detected outside of the high pressure apparatus. Annihilation rate ${ }^{2}$ and angular correletion ${ }^{3}$ measurements have been carried out for alkali metals under pressure, and the first positron annihilation (Doppler broadening) measurements in a diamond anvil cell were recentiy performed ${ }^{4}$ on $\mathrm{Fe}$ at pressures up to $40.0 \mathrm{GPa}$. 
In the present paper, we report calculations of the electron momentum density and partial annihilation rates (proportional to the two-photon momentum density) in polycrystalline compressed cesium. This material was chosen because its $s-d$ transition is particularly dramatic. At 1 atm, $C s$ is a free-electron metal; yet by only about $15 \mathrm{GPa}$, its single valence electron has been transfered entirely from the $6 \mathrm{~s}$ to the $5 d$ band. 5

\section{CALCULATIONS}

It is well known that electron-positron correlation enhances the electron density in the vicinity of the positron. Some success has been achieved, however, with a modified independent-particle model (IPM) in which electron states for the unperturbed crystal are used in the annihilation rate calculations along with enhancement factors to deal with the effects of the many-body electron-positron interaction. 6

In the IPM, modified to include an energy-dependent ${ }^{7}$ enhancement factor $c(\Sigma)$, the partial annihilation rate or probability per unit time and per unit volume of momentum space that an electron-positron annihilation will result in a pair of photons having a combined momentun of $\vec{p}$ is

$$
R(\vec{p})=r_{0}^{2} c \pi \frac{2}{\mathbb{N}} \underset{\overrightarrow{k j} \vec{G}}{\sum_{0 . c c}} e\left(E_{k j}\right)\left|A_{k j}(\vec{G})\right|^{2} \delta(\vec{p}-\vec{k}-\vec{G})
$$

where

$$
\vec{A}_{k j}=\frac{1}{\sqrt{\Omega}} \int_{\Omega} d \vec{r} e^{-i(\vec{k}+\vec{G}) \cdot \vec{r}} \Psi_{+}(\vec{r}) \psi_{k j}(\vec{r})
$$

Here, $r_{0}$ is the classical electron radius, $c$ is the speed of light, and $\mathbb{N}$ is the number of primitive cells in the crystal. The sums in Eq. (1) are over occupied electron states characterized by $\vec{k}$ in the first Brillouin zone and band Index $j$, and over reciprocal lattice vectors $\vec{G}$. The integral in Bq. (2) is over the primitive cell of volume $\Omega$ on which both the positron and elcctron wavefunctions, $\Psi_{+}(\vec{r})$ and $\psi_{\overrightarrow{k j}}(\vec{r})$, respectively, are normalized to unity.

The total annihilation rate $\lambda$ is the reciprocal of the positron lifetime, and is given by the integral of the partial anninilation rate $R(\vec{p})$ over all momentum space, Setting $r_{0}{ }^{2} c \pi=\left(E_{k_{j}}\right)=\psi_{+}(\vec{r})=1$ in Eqs. ( 1$)$ and $(2)$ converts $R(\vec{p})$ to the electron momentum density $p(\vec{p})$, which is customarily normalized so as to be unity for occupied, ideal, free-electron states, as will be done below.

Numerous calculations based on Eqs. (1) and (2) have been reported.6 As in recent work by Singh and Jarlborg, 8 the present effort used the linear muffin-tin orbitals method ${ }^{9}$ to obtain both the electron gtates for the unperturbed crystal, and the thermalized, $\vec{k}=0$, pure $s$, positron wavefunction. Our approach differs from that theirs, however, in calculation of the Fourier transform in Eq. (2). In the spirit of Andersen's combined correction, ${ }^{9}$ we approximate the integral in Eq. (2) by

$$
\left(\ldots \psi_{+} \psi_{k j}\right)_{\Omega}-\left(\ldots \psi_{+} \vec{\psi}_{k j}\right)_{s}+\psi_{+}(s)\left[\left(\ldots \vec{\psi}_{k j}\right)_{\Omega}-\left(\ldots \vec{\psi}_{k j}\right)_{s}\right]
$$

where $\left({ }_{\Omega}\right.$ and ()$^{\prime}$ indicate integration over the Wigner-Seitz polyhedra and sphere, respectively, $\Psi_{+}(S)$ is the value of the positron wavefunction 
at the Wigner-Seitz radius $s$, and $\tilde{v}_{k j}$ is the csual pseudowavefunction employed in the combined correction. To obtain the polycrystalline spherical average $R_{a v}(p)$ we use the tetrahedral method 10 to integrate over surface segments of fixed $p=h|\vec{k}+\vec{G}|$ for which $\vec{k}_{\vec{k} j}$ lies below the Fermi energy.

The present calculations were scalar relativistic (spin-orbit omitted), used the von Barth-Hedin exchange-correlation potential, 11 and treated all electrons self-consistently in generating the one-electron potentials. The 5s, 5p, and higher lying levels were included as band states in evaluating Eqs. (1) and (2), with angular momentum somponents through d character ratained. The Brillouin zone was sampled with 89 points in the irreducible wedge for the fcc structure. Each point was taken as the center of a small cube which was subdividad into 12 tetrahedra in obtaining the spherical average. The reciprocial lattice sum was truncated after 459 vectors. In on test calculation with 20 points per wedge and in another with 283 reciprocal lattice vectors, $R_{a v}(p)$ changed slightly and negligibly, respectively, showing convergence with the above choices.

\section{ELECTRON YOMENTUR DISTRIBUTION AND UNEMHANCED PARTIAL ANNIHILATION RATES}

Our results for the spherically averaged electron momentum density $P_{a y}(P)$ are shown in Fig. 1 for reduced volumes $V / V_{0}=1,0.418$, and 0.235 , where $v_{0}=795.5$ bohr $^{3} /$ atom. The corresponding pressures are $0,4.2$, and about $15 \mathrm{GPa}$, respectivley. At each volume, the solid curves give the total $P_{a v}(P)$, while the dashed curves show just the $5 s, 5 p$ core contribution. The trivial volume dependence has been removed by using the dimensionless momentum $p a / h$ where $a$ is the fcc lattice constant corresponding to each volume $\left(a^{3}=4 \mathrm{~V}\right)$, and $h$ is Planck's constant. Note that $\mathrm{pa} / \mathrm{h}=0.866,1$, and 1.732 at the $\mathrm{L}$ and $\mathrm{x}$ points and at the first reciprocal lattice vector, respectively.

For consistancy, we have reported all of our results for the fcc structure. While Cs exists in a bce phase at 1 atm, we find in bcc calculations ( 140 points per irreducible wedge, 459 reciprocal lattice

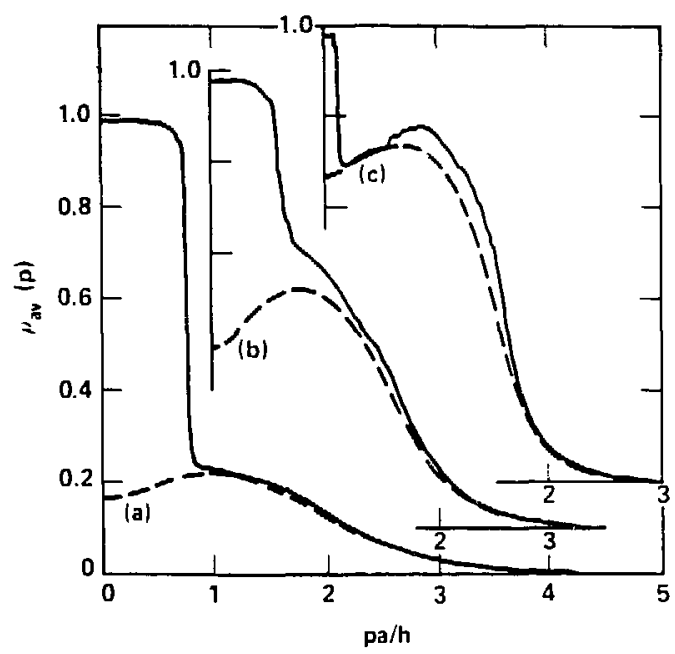

Fig. 1. Electron momentum density $\rho_{\mathrm{av}}(p)$ vs. dimensionless momentum $p a / h$ at a) $\left.v / v_{0}=1, b\right) v / v_{0}=0.418$, and $\left.c\right) v / v_{0}=0.235$. The solid and dashed lines show the total and core results, respectively. 
vectors) that the valence $\rho_{\mathrm{av}}(p)$ is nearly identical to that in Fig. $1(a)$, differing by less than about 17 of the solid curve, excep: in the small region of $\mathrm{pa} / \mathrm{h}=0.8-1.0$, where the differences are still less than 0.02 on the scale given in the figure. Cesium is fcc at $4.2 \mathrm{GPa}$, and appears to be either tetragonal or orthorhombic at $15 \mathrm{GPa}, 12$

The singie valence electron in 1-atm cs occupies sp-hybrid, 6s-band states near $\mathbf{k}=0$. This leads to the large valence density between the solid and dashed curves in Eig. $1(\mathrm{a})$ for $\mathrm{pa} / \mathrm{h}<0.78$, as well as the small spherical average of umklapp echos from about pa/h $=1.4$ to 2.2. Under compression, the rising $6 \mathrm{~s}$ band causes the Fermi momentum $P_{F}$ (where $P_{a y}(p)$ is nearly vertical) to decrease. The total valence density beneath $\mathrm{PF}$ drops from 0.73 , to 0.22 , and finally to 0.001 electrons in Figs. $\lambda(a), 1(b)$, and $1(c)$, respectively. Wear the conclusion of the s-d trarsition, Fig. I(c) shows most of the valence $\rho_{a v}(p)$ to be in the range $p a / h \approx 0.6-1.7$, which arises from $5 \mathrm{c}$ states near the $L$ and $X$ Brillouin zone boundery points including $a$ significant $\mathrm{x}$-point umklapp contribution around $\mathrm{pa} / \mathrm{h}=1.414$.

Even at 1 atm, there is significant overlap of the $5 s, 5 p$ cores in cs. By $15 \mathrm{GPa}$, the $5 p$ band is over $5 \mathrm{eV}$ wide, 5 and so it should be no surprise that momentum density in these increasingly more itinerant states should shift to lower values of $p$ as scen by the dashed curves in Fig. 3. Further reduction of volume be less than a factor of 2 results in a single, merged $5 s, 5 p$, $5 d$ valence band. 5

The partial annihilation rates $R_{a y}(p)$ are quite similar to Fig. 1; although, the relative importance of the $5 s, 5 p$ cores is reduced given the positron-nucleus repulsion. Given this similarity, we plot these results in an altersate form in Fig. 2, namely $4 \pi p^{2} R_{a v}(p)$, so that the area is just the total annihilation rate $\lambda$. The counterpart of the $6 \mathrm{~s}$-band free-electron plateau in Fig. $1(a)$ is now the initial $\mathrm{p}^{2}$ rise at small $p$ in Fig. 2(a), followed, of course, by the rapid drop at $P F$. This characteristic structure is greatly reduced in Fig. $2(b)$ at $V / V_{0}=0.418$, and is entirely gone by Fig. $3(c)$ at $v / V_{0}=0.235$ as the s-d transition is nearly completed. The growing partial annihilation rate from the newly occupied $5 d-1$ ike $:$ tates can be seen as

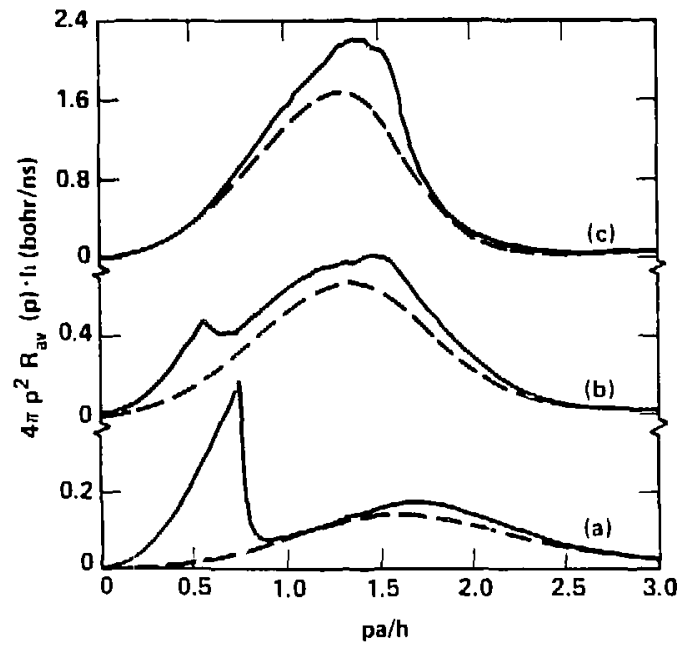

Fig. 2. Partial annihilation rates $4 \pi p^{2} R_{a v}(p)$ vs. dimensionless momentum $p a / h$ a.t a) $\left.V / v_{0}=1, b\right) v / v_{0}=0.418$, and c) $V / V_{0}=0.235$. The solid and dashed lines show the total and core results, respactively. No enhancement was used. 


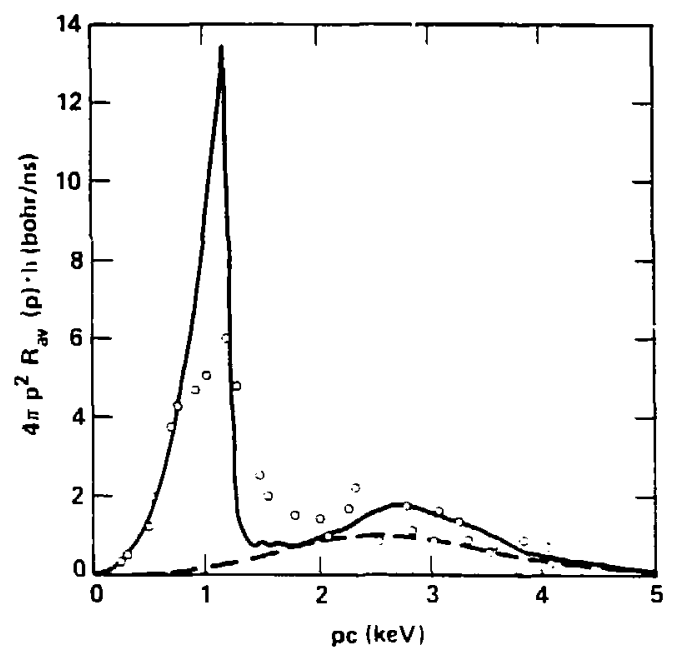

Fig. 3. Enhanced partial annihilation rate $4 \pi p^{2} R_{a v}(p)$ vs. $p c$ at $V / V_{O}=1$. The solid and dashed lines show the total and core results, respectively. The data points are from Ref. 14, and were normalized to agree with the solid curve at small momentum.

the increasing separation between the solid and dashed curves in the vicinity of $\mathrm{pa} / \mathrm{h} \approx 1.4$.

\section{ENHANCED PARTIAL ANNIHILATION RATE}

The question of what enhancement factor should be used for a given metal is still under study.13 While it is beyond the scope of this preliminary work to deal with this question in any depth, we do provide in Fig. 3 an enhanced counterpart of Fig. 2(a) plotted against pe to give more familiar units. The factor $\varepsilon(\varepsilon)$ in Eq. ( 1 ) was taken from a recent paper by Sob who specified coeffients for $G s$ at 1 atm. 7

Without enhancement, our results in Fig. 2(a) yield total annihilation rates of 0.093 and $0.068 \mathrm{~ns}^{-1}$ for core and valence electrons, respectively. With the enhancement, the curves in Fig. 3 yield values of 0.663 and $1.668 \mathrm{~ns}^{-1}$ for core and valence electrons, respectivley, in good agreement with Sob's analysis. The sum of core and valence contributions, $2.33 \mathrm{~ns}^{-1}$, is within $3 \%$ of the experimental annihilation rate. 2,7 It should be noted that the extremely low electron density in $G$ s leads to both this unusually low annihilation rate as well as the very large enhancement in this material.

The data points in Fig. 3 are taken from a 1957 paper by stewart. 14 They result from his differentiation of (instrumentalresolution corrected) long-slit angular correlation data, scaled by us to agree with the solid curve at small $\mathrm{p}$. The disagreement between theory and experiment near the $\mathrm{PFC}=1.25 \mathrm{keV}$ is consistent with recent work by Chakraborty 15 which indicates that the Kahana-based enhancement used in Ref. 7 is likely to be too large just below $P_{F}$ and too small just above $\mathrm{PF}_{F}$. Although there is considerable scatter in the range $p c \approx 2-4$. kev, there is clear evidence for at least a shuulder to the main peak near pc $\approx 1 \mathrm{keV}$ in agreement with the present calculations. 


\section{CONGLUSIONS}

We suggest that the gross features of the s-d transition in compressed Cs should be clearly visible in high-pressure positron annihilation measurements even at resolutions obtainable with the Doppler-broadenirg technique. Specifically, the 6s-band dominated peak $(p a / h \approx 0.3-0.7)$ seen in $4 \pi p^{2} R_{a v}$ at 1 atm should decay under compression, as the stoulder region evolves into a new, broad, much larger peak ( $\mathrm{pa} / \mathrm{h} \approx 0.8-1.7$ ) arising out of core and $5 \mathrm{~d}$ zoneboundary contributions. Beyond these qualitative features, a quantitative comparison with experiment night prove highly informative in regard to the nature of enhancement effects in a range where core levels evolve into broad-band valence states.

\section{ACKNOWLEDGMENTS}

Part of this work (that of A.K.M.) was performed under the auspices of the U. S. Deparment of Energy by Lawrence Livermore National Laboratory under Contract No. W-7405-ESG-48, and part (that of H.L.S.) was supported by the Niels Bohr Foundation through the Royal Danish Academy of Sciences and Letters.

\section{REFERENCES}

1. See., e.8., H. Ross and A. K. McMahan, Phys. Rev. B 26, 4088, 1982; and H. L. Skriver, Phys. Rev. B 31, 1909 (1985), and references therein.

2. I. K. MacKenzie, R. LeBlanc, and B. T. A. McKee, Phys. Rev. Lett. 27. 580 (1971)

3. D. R. Gustafson and J. D. Willenberg, Phys. Rev. B 13, 5193 (1976),

4. H. B. Radousky, R. L, Reichlin and R. H. Howell, J, Phys. (Paris), C8, 369 (1984).

5. A. K. Mckahan, Phys. Rev, B 29, 5982 (1984).

6. See, e.B., P. E. Mijnarends in Positrons in Solids, edited by $P$. Häutojarvi (Springer, Berîin, 1979), p. 25.

7. For 1-atm Cs, Sob [M. Sob, Solid state Commun. 54, 249 (1985)] suggests $a=16.24, b / a=0.5$, and $c / a=0.4$ in his $E q$. (7) for the valence enhancement $\varepsilon_{v}(E)$, and a constant core enhancement factor of 7.1 .

8. A. K. Singh and T. Jarlborg, J. Phys, F 15, 727 (1985).

9. O. K. Andersen, Phys. Rev. B 12, 3060 (1975); H. L. Skriver, The LYTO Method (Springer, Berlin, 1984).

10. 0. Jepsen and 0. K. Andersen, Solid State Commun. 9, 1763 (1971). See last column in Table 1.

11. U. von Barth and L. Hedin, J. Phys. C 1629 (1972).

12. K. Takemura, S. Minomura, and 0 . Shimomura Phys. Rev. Lett. 49, 1772 (1982); $K$. Takemura and $K$. Syassen iti Proc. of the Int. Symp. on Solid State Physcis under Pressure, edited by B.I.N. Okai (Academic Press of Japan, Tokyo, 1984), and references therein.

13. See, e.g., Posilron Annihilation, edited by P. G. Coleman, S. C. Sharma, and L. M. Diana (North-Holland, Amsterdam, 1982).

14. A. T. Stewart, Can. J. Phys. 35,168 (1957).

15. B. Chakraborty, Phys. Rev. B 24,7423 (1981). 